\title{
On the Convergence of Ritz-Galerkin's Method
}

\author{
By
}

Tetsuhiko MryoshI

\section{§1. Introduction}

The present paper is concerned with Ritz-Galerkin's method to solve approximately the boundary value problem:

$$
\begin{gathered}
\frac{\partial}{\partial x}\left(a \frac{\partial u}{\partial x}\right)+\frac{\partial}{\partial y}\left(b \frac{\partial u}{\partial y}\right)-c u=f \text { in } D \\
\left.u\right|_{r}=0
\end{gathered}
$$

where $D$ is a bounded domain in the $(x, y)$-plane, $\Gamma$ is the boundary of $D$, and $a(>0), b(>0), c(\geq 0), f$ are smooth functions defined on $\bar{D}$. In Ritz-Galerkin's method, first we choose a system of linearly independent functions $\left\{\varphi_{k}\right\}$ such that they satisfy the given homogeneous boundary condition and they are dense in a function space containing the exact solution of the above boundary value problem, and next we seek the $m$-th approximation $u_{m}$ in the form

$$
u_{m}=\sum_{k=1}^{m} a_{k} \varphi_{k}
$$

calculating the coefficients $\left\{a_{k}\right\}$ by solving a system of equations

$$
\sum_{k=1}^{m} \alpha_{k, s} a_{k}+\beta_{s}=0 \quad(s=1,2, \cdots, m),
$$

where

$$
\left\{\begin{array}{l}
\alpha_{k, s}=\alpha_{s, k}=\iint_{D}\left[a \frac{\partial \varphi_{k}}{\partial x} \frac{\partial \varphi_{s}}{\partial x}+b \frac{\partial \varphi_{k}}{\partial y} \frac{\partial \varphi_{s}}{\partial y}+c \varphi_{k} \varphi_{s}\right] d x d y \\
\beta_{s}=\iint_{D} f \cdot \varphi_{s} d x d y \quad(k, s=1,2, \cdots, m)
\end{array}\right.
$$

Received March 14, 1968. 
The system of equation (1.3) is called the determining equation of Ritz-Galerkin's method.

Ritz-Galerkin's method was studied by many people, sometimes by the use of classical techniques as in [1], [2], and sometimes by the use of functional analysis as in [3], [4]. However Ritz-Galerkin's method does not seem to be frequently practised on a modern highspeed digital computer. The main reason for this seems to be in the difficulty in finding a system of functions $\left\{\varphi_{k}\right\}$ having the desired property. In [1], Kantorovich presents a method to construct such a system of functions and proves the uniform convergence of the approximate solution obtained from this system of functions. However it is done only for the domain strongly restricted. In [2], Harrik proves the uniform convergence of the approximate solution obtained by Ritz-Galerkin's method for the domain with smooth boundary, but he does not present any method to construct a system of functions $\left\{\varphi_{k}\right\}$ adapt for such domain.

In the present paper, the author will present a method to find a system of functions $\left\{\varphi_{k}\right\}$ adapt for some kind of domains, say, $R$-type domain, and further he will prove the uniform convergence of the approximate solution obtained by Ritz-Galerkin's method for the domain with great generality. Numerical examples are to appear in the subsequent paper. The author acknowledges the encouragement and the suggestions of Professor M. Urabe rendered during the preparation of the present paper.

\section{§2. A Method to Find a System of Functions $\left\{\varphi_{\boldsymbol{k}}\right\}$ Necessary for Ritz-Galerkin's Method}

To begin with, we seek function $\omega(x, y)$ which satisfies the given homogeneous boundary condition together with some additional conditions. Let $D$ be a given bounded domain in the $(x, y)$-plane. The conditions which we request for function $\omega(x, y)$, are then as follows:

( I ) $\omega(x, y)=0$ on boundary $\Gamma$ of $D$, and $\omega(x, y)>0$ in the interior of $D$.

(II) $\omega(x, y)$ is continuous and has bounded first derivatives in $\bar{D}$. 
In addition, the first derivatives are continuous in $D$ and have bounded piecewise continuous derivatives in any closed subregion of $D$.

(III) There are five positive constants $\bar{\delta}, \lambda_{1}, \lambda_{2}, \bar{C}_{1}, \bar{C}_{2}$ such that

$$
\begin{aligned}
& \left|\partial_{i j}^{2} \omega(x, y)\right| \leq \overline{C_{1}} \rho(x, y)^{-\lambda_{1}} \quad(i, j=1,2) \\
& \omega(x, y) \geq \overline{C_{2} \rho}(x, y)^{\lambda_{2}}
\end{aligned}
$$

in $D$ for $\rho(x, y) \equiv \operatorname{dist}((x, y), \Gamma) \geq \bar{\delta}$. Here $\partial_{i j}^{2} \omega$ denotes $\partial^{2} \omega / \partial x_{i} \partial x_{j}$ where $x_{1}=x$ and $x_{2}=y$.

If we can find a function $\omega(x, y)$ satisfying the above conditions, then using such $\omega(x, y)$, we consider the system of functions

$$
\left\{\omega(x, y) x^{k} y^{s}\right\} \quad(k, s=0,1,2, \cdots),
$$

which, as will be shown later, is indeed one of the desired system of functions $\left\{\varphi_{k}\right\}$. Hence the problem to find a system of functions necessary for Ritz-Galerkin's method reduces to find a function $\omega(x, y)$ satisfying the above conditions.

Now, we shall show the method to construct a function $\omega(x, y)$ satisfying the above conditions for some kind of domains.

$1^{\circ}$. The domain with algebraic boundary curves.

Let

$$
\prod_{i=1}^{r} p_{i}(x, y)=0
$$

be the equation of the boundary curve, where $p_{i}(x, y)$ are polynomials of $x$ and $y$ such that $p_{i}(x, y) \neq 0$ for $(x, y) \in D$. Then the desired $\omega(x, y)$ can be given by

$$
\omega(x, y)=\prod_{i=1}^{r} p_{i}(x, y) .
$$

A circle, ellipse, polygon and the domain composed of these are the examples.

$2^{\circ}$. R-type domain. By $R$-type domain, is meant the domain composed of a finite number of rectangles whose sides are all parallel to either of the coordinate axes. For the $R$-type domain, neither the convexity nor the simple connectedness is assumed. 
Before considering the general $R$-type domain, let us consider the simple case where the domain $D$ is a $L$-type domain, that is, the domain composed of three adjacent rectangles connected as in Fig. 1. For such L-type domain, let us construct a function $\omega(x, y)$ such that

(a) $\omega(x, y)$ satisfies the conditions (I), (II), (III);

(b) $\omega_{x}(x, y)=0$ on BGL, CHO, DIMQT and arc HM, $\omega_{y}(x, y)=0$ on $\mathrm{PQR}, \mathrm{OMN}, \mathrm{FGHIJ}$ and on arc HM.

Function $\omega(x, y)$ satisfying the above conditions can be easily found as illustrated below with two examples in the case where the rectangles composing the domain are all unit squares.

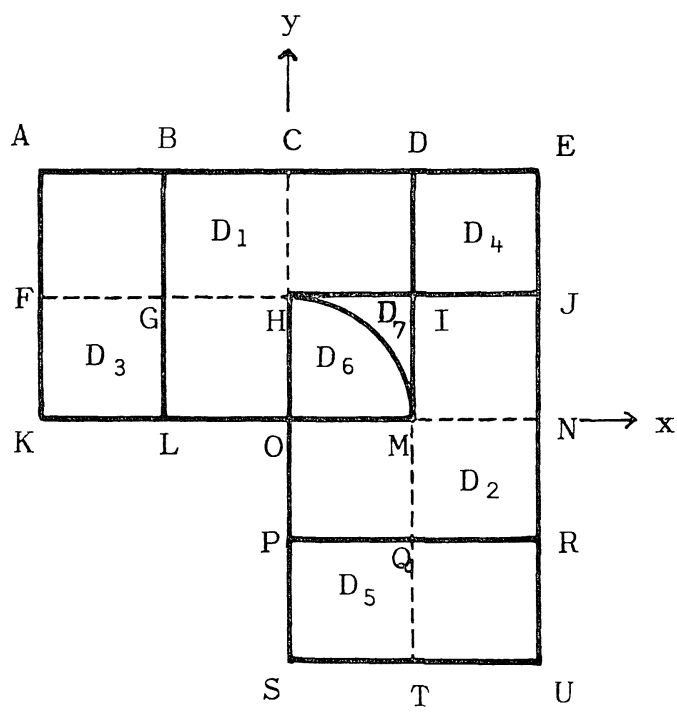

Fig. 1

Example 1.

$$
\omega(x, y)= \begin{cases}4\left(y-y^{2}\right) & \text { in } \mathrm{D}_{1} \\ 4\left(x-x^{2}\right) & \text { in } \mathrm{D}_{2} \\ 16\left(x^{2}+x\right)\left(y^{2}-y\right) & \text { in } \mathrm{D}_{3} \\ 16\left(x^{2}-x\right)\left(y^{2}-y\right) & \text { in } \mathrm{D}_{4} \\ 16\left(x^{2}-x\right)\left(y^{2}+y\right) & \text { in } \mathrm{D}_{5} \\ 4\left[\sqrt{x^{2}+y^{2}}-\left(x^{2}+y^{2}\right)\right] & \text { in } \mathrm{D}_{6} \\ 1 & \text { in } \mathrm{D}_{7}\end{cases}
$$




\section{Example 2.}

$$
\omega(x, y)= \begin{cases}16\left(\frac{1}{2} y^{2}-y^{4}\right)\left(4 x^{2}-1\right)^{2}+4\left(y-y^{2}\right)\left[1-\left(4 x^{2}-1\right)^{2}\right] & \text { in } \text { GHOL } \\ 4\left(y-y^{2}\right) & \text { in } \text { BDIG } \\ 16\left(\frac{1}{2} x^{2}-x^{4}\right)\left(4 y^{2}-1\right)^{2}+4\left(x-x^{2}\right)\left[1-\left(4 y^{2}-1\right)^{2}\right] & \text { in } \text { OMQP } \\ 4\left(x-x^{2}\right) & \text { in IJRQ } \\ 16\left(x^{2}+x\right)\left(y^{2}-y\right) & \text { in } \mathrm{D}_{3} \\ 16\left(x^{2}-x\right)\left(y^{2}-y\right) & \text { in } \mathrm{D}_{4} \\ 16\left(x^{2}-x\right)\left(y^{2}+y\right) & \text { in } \mathrm{D}_{5} \\ -16\left(x^{2}+y^{2}\right)\left(x^{2}+y^{2}-\frac{1}{2}\right) & \text { in } \mathrm{D}_{6} \\ 1 & \text { in } \mathrm{D}_{7}\end{cases}
$$

Note that in Example 1, function $\omega(x, y)$ is continuously differentiable in $D$, while in Example 2, it is continuously differentiable in $\bar{D}$.

Now we shall consider the general $R$-type domain. Let us note that in Example 1, function $\omega(x, y)$ is constructed by linking the functions of the following five types:

$$
\begin{array}{cc}
4\left(y-y^{2}\right) & \text { in } \mathrm{D}_{1}, \\
16\left(x^{2}+x\right)\left(y^{2}-y\right) & \text { in } \mathrm{D}_{3}, \\
16\left(x^{2}-x\right)\left(y^{2}-y\right) & \text { in } \mathrm{D}_{4}, \\
1 & \text { in } \mathrm{D}_{7}, \\
4\left[\sqrt{x^{2}+y^{2}}-\left(x^{2}+y^{2}\right)\right] & \text { in } \mathrm{D}_{6} .
\end{array}
$$

In Example 2, function $\omega(x, y)$ is similarly constructed by linking the functions of the following six types:
(1) $4\left(y-y^{2}\right)$
in $\mathrm{BDIG}$,
(2) $16\left(x^{2}+x\right)\left(y^{2}-y\right)$
in $\mathrm{D}_{3}$,
(3) $16\left(x^{2}-x\right)\left(y^{2}-y\right)$
in $\mathrm{D}_{4}$,
(4) 1
in $\mathrm{D}_{7}$,
(5) $-16\left(x^{2}+y^{2}\right)\left(x^{2}+y^{2}-\frac{1}{2}\right)$ in $\mathrm{D}_{6}$,
(6) $16\left(\frac{1}{2} y^{2}-y^{4}\right)\left(4 x^{2}-1\right)^{2}+4\left(y-y^{2}\right)\left[1-\left(4 x^{2}-1\right)^{2}\right] \quad$ in GHOL. 
Then we see without difficulty that the desired function $\omega(x, y)$ can be constructed for the general $R$-type domain by linking the functions derived from those of the above types by simple transformations, that is, expansion, contraction, rotation and translation. Moreover such construction of function $\omega(x, y)$ can be done on a computer without much difficulty.

The second derivatives of function $\omega(x, y)$ constructed in the above way are not continuous in $D$, but, if necessary, we can construct without much difficulty the function $\omega(x, y)$ having continuous second derivatives by the similar method.

\section{§3. Notations and Function Spaces}

In the present section, the notations which will be used frequently in the later secitons will be explained and the function spaces which will be necessary for subsequent discussions will be introduced.

$1^{\circ} . \quad \partial_{i} u(i=1,2)$ means $\partial u / \partial x_{i}$ and $\partial_{i j}^{2} u(i, j=1,2)$ means $\partial^{2} u / \partial x_{i} \partial x_{j}$, where $x_{1}=x$ and $x_{2}=y$. Sometimes notations $\partial_{x} u$ and $\partial_{y} u$ will be also used for $\partial u / \partial x$ and $\partial u / \partial y$ respectively.

$2^{\circ}$. By a polynomial of degree $m$, we mean the polynomial of the form

$$
\sum_{k, s=0}^{m} a_{k, s} x^{k} y^{s}
$$

and such a polynomial will be denoted by the notation $p_{m}(x, y)$.

$3^{\circ}$. $T_{k}(x)$ denotes a Chebyshev polynomial of degree $k$, that is,

$$
T_{k}(x)=\cos k\left(\cos ^{-1} x\right) .
$$

In what follows, we suppose $D$ is a bounded domain in the $(x, y)$ plane and all functions are real-valued.

$4^{\circ}$. By $\dot{C}^{k}(D)$, we denote the space consisting of functions which are defined on $D$, vanish on the boundary of $D$ and have continuous $k$-th derivatives in $D$. 
$5^{\circ}$. By $\stackrel{\circ}{C}^{k}(D)$, we denote the subspace of $\dot{C}^{k}(D)$ consisting of all functions with supports in $D$.

$6^{\circ}$. By $\stackrel{\circ}{W}_{2}^{1}(D)$, we denote the completion of $\stackrel{\circ}{C}^{\infty}(D)$ under the norm $\|\cdot\|_{1, L^{2}(D)}$ defined by

$$
\|u\|_{1, L^{2}(D)}^{2}=\|u\|_{L^{2}(D)}^{2}+\left\|\partial_{x} u\right\|_{L^{2}(D)}^{2}+\left\|\partial_{y} u\right\|_{L^{2}(D)}^{2} .
$$

Lastly we consider a square $S$ whose sides are $x= \pm 1,|y| \leq 1$ and $|x| \leq 1, y= \pm 1$.

$7^{\circ}$. By $L_{T}^{2}(S)$, we denote the space consisting of functions such that they are measurable with respect to the Lebesgue-Stieltjes measure generated by the function $\varphi(x, y)=\cos ^{-1} x \cos ^{-1} y$ and moreover their Lebesgue-Stieltjes integral

$$
\iint_{s} u^{2}(x, y) d \varphi(x, y)
$$

is finite. In the space $L_{T}^{2}(S)$, the norm will be introduced by

$$
\|u\|_{T}=(u, u)_{T}^{1 / 2} \equiv\left[\iint_{S} u^{2}(x, y) d \varphi(x, y)\right]^{1 / 2},
$$

which is clearly equal to

$$
\left.\left[\iint_{s} \frac{u^{2}(x, y)}{\sqrt{1-x^{2}} \sqrt{1-y^{2}}} d x d y\right]^{1 / 2}=\left[\int_{0}^{\pi} \int_{0}^{\pi} u^{2}(\cos \theta, \cos \tilde{\theta}) d \theta d \tilde{\theta}\right)\right]^{1 / 2} .
$$

Evidently the space $L_{T}^{2}(S)$ is a Hilbert space if the inner product is introduced by

$$
(u, v)_{T}=\iint_{s} u(x, y) v(x, y) d \varphi(x, y)
$$

\section{$\S 4$. Theoretical Background of Ritz-Galerkin's Method}

It is known that the boundary value problem (1.1) has a unique solution $\bar{u}(x, y) \in \dot{C}^{2}(D) \cap \stackrel{\circ}{W}_{2}^{1}(D)$ satisfying the equality

$$
\left(a \frac{\partial \bar{u}}{\partial x}, \frac{\partial \varphi}{\partial x}\right)_{L^{2}(D)}+\left(b \frac{\partial \bar{u}}{\partial y}, \frac{\partial \varphi}{\partial y}\right)_{L^{2}(D)}+(c \bar{u}, \varphi)_{L^{2}(D)}=-(f, \varphi)_{L^{2}(D)}
$$

for any $\varphi(x, y)$ in $\stackrel{\circ}{W}_{2}^{1}(D)$ if the boundary of $D$ satisfies a certain condition, say, Poincaré condition (see [7]). In what follows, the above 
condition on $D$ will be called condition $(A)$.

Now, consider the functional

$$
I(u)=\iint_{D}\left[a\left(\frac{\partial u}{\partial x}\right)^{2}+b\left(\frac{\partial u}{\partial y}\right)^{2}+c u^{2}+2 f u\right] d x d y
$$

defined for $u \in W_{2}^{1}(D)$, and let $\hat{u}(x, y)$ be an arbitrary function belonging to $\stackrel{\circ}{W}_{2}^{1}(D)$. Then, by (4.1), for $\eta=\hat{u}-\bar{u}$, we have

$$
\begin{aligned}
& I(\hat{u})-I(\bar{u}) \\
= & \iint_{D}\left[a\left(\frac{\partial(\bar{u}+\eta)}{\partial x}\right)^{2}+b\left(\frac{\partial(\bar{u}+\eta)}{\partial y}\right)^{2}+c(\bar{u}+\eta)^{2}+2 f(\bar{u}+\eta)\right] d x d y \\
& -\iint_{D}\left[a\left(\frac{\partial \bar{u}}{\partial x}\right)^{2}+b\left(\frac{\partial \bar{u}}{\partial y}\right)^{2}+c \bar{u}^{2}+2 f \bar{u}\right] d x d y \\
= & \iint_{D}\left[a\left(\frac{\partial \eta}{\partial x}\right)^{2}+b\left(\frac{\partial \eta}{\partial y}\right)^{2}+c \eta^{2}\right] d x d y \geq 0 .
\end{aligned}
$$

The above inequality shows that the solution of the boundary value problem (1.1) minimizes the functional $I(u)$ in the space $\stackrel{\circ}{W}_{2}^{1}(D)$ provided the boundary of the domain $D$ satisfies condition $(A)$.

Now let $\left\{\varphi_{k}(x, y)\right\}(k=1,2, \cdots, m)$ be a system of linearly independent functions belonging to $\dot{C}^{1}(D) \cap \stackrel{\circ}{W}_{2}^{1}(D)$ and put

$$
u_{m}(x, y)=\sum_{k=1}^{m} a_{k} \varphi_{k}(x, y) .
$$

Then from (4.2), by (1.4), we see that

$$
I\left(u_{m}(x, y)\right)=\sum_{k, s=1}^{m} \alpha_{k, s} a_{k} a_{s}+2 \sum_{s=1}^{m} \beta_{s} a_{s} .
$$

Since

$$
\sum_{k, s=1}^{m} \alpha_{k, s} a_{k} a_{s}=\iint_{D}\left[\left(a\left(\frac{\partial u_{m}}{\partial x}\right)^{2}+b\left(\frac{\partial u_{m}}{\partial y}\right)^{2}+c u_{m}^{2}\right] d x d y,\right.
$$

the quadratic form $\sum_{k, s=1}^{m} \alpha_{k, s} a_{k} a_{s}$ is positive definite. Then $I\left(u_{m}\right)$ as a second degree polynomial of $\left\{a_{k}\right\}(k=1,2, \cdots, m)$ assumes a minimum value when and only when

$$
\sum_{k=1}^{n} \alpha_{k, s} a_{k}+\beta_{s}=0 \quad(s=1,2, \cdots, m) .
$$

This equality coincides with determining equation (1.3) of RitzGalerkin's method. This means that $I\left(u_{m}\right)$ assumes a minimum value 
when $u_{m}$ is the $m$-th approximation obtained by Ritz-Galerkin's method. Now the quadratic form $\sum_{k, s=1}^{m} \alpha_{k, s} a_{k} a_{s}$ is positive definite, therefore clearly $\operatorname{det}\left(\alpha_{k, s}\right) \neq 0$. This implies the determining equation has always a unique solution. in other words, in Ritz-Galerkin's method, the $m$-th approximation $u_{m}(x, y)$ can be always determined uniquely for every $m$.

Let $S_{m}$ be a linear manifold generated by a system of functions $\varphi_{k}(x, y)(k=1,2, \cdots, m)$ and $\hat{u}_{m}$ be an arbitrary function belonging to $S_{m}$. Let $\bar{u}(x, y)$ be the exact solution of our boundary value problem and $u_{m}(x, y)$ be the $m$-th approximation obtained by Ritz-Galerkin's method. Then, for $\hat{\eta}_{m}=\hat{u}_{m}-\bar{u}$ and $\eta_{m}=u_{m}-\bar{u}$, by (4.3), we have

$$
\begin{aligned}
& \iint_{D}\left[a\left(\frac{\partial \hat{\eta}_{m}}{\partial x}\right)^{2}+b\left(\frac{\partial \hat{\gamma}_{m}}{\partial y}\right)^{2}+c \hat{\eta}_{m}^{2}\right] d x d y \\
& \quad=I\left(\hat{u}_{m}\right)-I(\bar{u}) \\
& \quad \geq I\left(u_{m}\right)-I(\bar{u}) \\
& \quad=\iint_{D}\left[a\left(\frac{\partial \eta_{m}}{\partial x}\right)^{2}+b\left(\frac{\partial \eta_{m}}{\partial y}\right)^{2}+c \eta_{m}^{2}\right] d x d y .
\end{aligned}
$$

However, as readily seen,

$$
\|\eta\|_{J}=\left[\iint_{D}\left\{a\left(\frac{\partial \eta}{\partial x}\right)^{2}+b\left(\frac{\partial \eta}{\partial y}\right)^{2}+c \eta^{2}\right\} d x d y\right]^{1 / 2}
$$

can be regarded as a norm of $\eta \in \stackrel{\circ}{W}_{2}^{1}(D)$. Then inequality (4.8) means that

$$
\left\|u_{m}-\bar{u}\right\|_{J} \leq\left\|\hat{u}_{m}-\bar{u}\right\|_{J}
$$

Since $\hat{u}_{m}$ is an arbitrary function belonging to $S_{m}$, the above inequality implies that $u_{m}$ is the best approximation in $S_{m}$ in the norm $\|\cdot\|_{J}$.

It is expected that the approximate solution $u_{m}(x, y)$ converges uniformly to the exact solution $\bar{u}(x, y)$ if the difference $\left\|u_{m}-\bar{u}\right\|_{J}$ tends to zero as $m \rightarrow \infty$. The subsequent sections are concerned with this convergence problem and our discussions are based on the following theorem which follows immediately from Lemma 1 in p. 338 of [1].

Theorem 4.1. Let $T$ be the boundary of the domain $D$ and 
$\eta(x, y)$ be a function belonging to $C^{\mathrm{i}}(D)$ satisfying the following conditions:

$$
\begin{aligned}
& J(\eta)=\iint_{D}\left[\left(\frac{\partial \eta}{\partial x}\right)^{2}+\left(\frac{\partial \eta}{\partial y}\right)^{2}\right] d x d y \leq \varepsilon, \\
& \int_{l_{\phi} \cap D}\left(\frac{d \eta}{d s}\right)^{2} d s \leq K_{0}
\end{aligned}
$$

for any point $p$ lying in $D$, where $l_{p}$ is a suitable line segment of fixed length $L_{0}$ centered at point $p$, and $K_{0}$ is a constant independent of the point $p$. Then for $(x, y) \in D$, we have

$$
|\eta(x, y)| \leq \hat{C}_{1} \sqrt{\varepsilon \log \frac{d K_{0}}{\varepsilon}}+\hat{C}_{2} \sqrt{\varepsilon}
$$

if $2 L_{0} \geq \varepsilon / K_{0}$ and $d K_{0}>\varepsilon$, and

$$
|\eta(x, y)| \leq \widehat{C}_{3} \sqrt{\varepsilon}
$$

otherwise. Here $d$ is the diameter of the domain $D$ and

$$
\begin{aligned}
& \widehat{C}_{1}=\frac{1}{2}+\frac{\pi}{6}, \quad \widehat{C_{2}}=\frac{2}{\sqrt{3}}+\frac{1}{\sqrt{2}} . \\
& \widehat{C}_{3}=\max \left[1+\frac{\pi}{12} \log \frac{d}{2 L_{0}}+\frac{2}{\sqrt{3}}+\frac{1}{\sqrt{2}}, 1+\frac{2}{\sqrt{3}}+\frac{1}{\sqrt{2}}\right] .
\end{aligned}
$$

\section{$\S 5$. Some Properties of Double Chebyshev Series}

In the present section, we shall derive some properties of double Chebyshev series which will be used later for proving the uniform convergence of the approximate solution obtained by Ritz-Galerkin's method.

For simplicity, let us suppose that the closure of domain $D$ lies in the interior of the square $S$ whose sides are $x= \pm 1,|y| \leq 1$ and $|x| \leq 1, y \pm 1$. Then clearly $\operatorname{dist}(\Gamma, \partial S)>0$, where $\Gamma$ is the boundary of $D$ and $\partial S$ is the boundary of $S$.

Let $u, v$ be arbitrary functions belonging to $L_{T}^{2}(S)$. Then by the inner product

$$
(u, v)=\int_{0}^{\pi} \int_{0}^{\pi} u(\cos \theta, \cos \tilde{\theta}) v(\cos \theta, \cos \tilde{\theta}) d \theta d \tilde{\theta},
$$


the space $H \equiv\left\{u(\cos \theta, \cos \tilde{\theta}) ; u \in L_{T}^{2}(S)\right\}$ can be regarded as a Hilbert space consisting of functions defined on $0 \leq \theta, \tilde{\theta} \leq \pi$. In such a Hilbert space $H$, the system of functions

$$
\left\{\frac{e_{k, s}}{\pi} \cos k \theta \cos s \tilde{\theta}\right\} \quad(k, s=0,1,2 \cdots)
$$

is clearly a complete orthonormal system, where

$$
e_{k, s}=\left\{\begin{array}{cll}
1 & \text { for } & k=s=0 \\
\sqrt{2} & \text { for } & k s=0, k \neq s, \\
2 & \text { for } & k, s \neq 0 .
\end{array}\right.
$$

Let

$$
u(\cos \theta, \cos \tilde{\theta}) \sim \sum_{k, s=0}^{\infty} e_{k, s} A_{k, s} \cos k \theta \cos s \tilde{\theta}
$$

be the Fourier series of $u(\cos \theta, \cos \tilde{\theta}) \in H$, then clearly the Fourier coefficient $A_{k, s}$ are given by

$$
A_{k, s}=\frac{e_{k, s}}{\pi^{2}} \int_{0}^{\pi} \int_{0}^{\pi} u(\cos \theta, \cos \tilde{\theta}) \cos k \theta \cos s \tilde{\theta} d \theta d \tilde{\theta}
$$

and we have Parseval's equality of the following form

$$
\int_{0}^{\pi} \int_{0}^{\pi} u^{2}(\cos \theta, \cos \tilde{\theta}) d \theta d \tilde{\theta}=\pi^{2} \sum_{k, s=0}^{\infty} A_{k, s}^{2} .
$$

By (3.2) and (3.6), Fourier series (5.4) and Parseval's equality (5.6) can be written respectively as follows:

$$
\begin{aligned}
& u(x, y) \sim \sum_{k, s=0}^{\infty} e_{k, s} A_{k, s} T_{k}(x) T_{s}(y) \\
& \|u\|_{T}^{2}=\pi^{2} \sum_{k, s=0}^{\infty} A_{k, s}^{2} .
\end{aligned}
$$

Hereafter the series in the right-hand side of (5.7) will be called the (double) Chebyshev series and its coefficient $A_{k, s}$ will be called the Chebyshev coefficient of function $u(x, y)$. Equality (5.8) valid for Chebyshev series (5.7) will be hereafter called Parseval's equality for Chebyshev series.

Now, we shall derive properties of double Chebyshev series necessary for later discussions following the method used by Urabe [5] for simple Chebyshev series. 
Theorem 5.1. Suppose that $u(x, y)$ is continuous and has piecewise continuous first derivatives in $\bar{S}$. Let

$$
\begin{aligned}
& u(x, y) \sim \sum_{k, s=0}^{\infty} e_{k, s} A_{k, s} T_{k}(x) T_{s}(y), \\
& u_{x}(x, y) \sim \sum_{k, s=0}^{\infty} e_{k, s} A_{k, s}^{\prime} T_{k}(x) T_{s}(y)
\end{aligned}
$$

be the Chebyshev series of $u(x, y)$ and $u_{x}(x, y)$ respectively, then it holds that

$$
\begin{aligned}
& A_{k, s}= \frac{1}{2 k}\left(e_{k-1} A_{k-1, s}^{\prime}-A_{k+1, s}^{\prime}\right) \quad(k \geq 1, s \geq 0), \\
& A_{k, s}^{\prime}=\frac{2}{e_{k}}\left[(k+1) A_{k+1, s}+(k+3) A_{k+3, s}+(k+5) A_{k+5, s}+\cdots\right], \\
& \quad(k, s \geq 0)
\end{aligned}
$$

where

$$
e_{k}=\left\{\begin{array}{cl}
\sqrt{2} & \text { for } k=0 \\
1 & \text { otherwise. }
\end{array}\right.
$$

Similar relations hold also for Chebyshev coefficients of $u_{y}$ and $u$.

Proof. Equation (5.11) can be easily proved by substituting the formula (5.5) for $A_{k-1, s}^{\prime}$ and $A_{k+1, s}^{\prime}$. Equality (5.12) readily follows from (5.11) by Parseval's equality.

Q. E. D.

Now, for function $u(x, y) \in L_{T}^{2}(S)$, we consider an operator $P_{m, n}$ such that

$$
P_{m, n} u(x, y)=\sum_{k=0}^{m} \sum_{s=0}^{n} e_{k, s} A_{k, s} T_{k}(x) T_{s}(y)
$$

where $A_{k, s}$ are the Chebyshev coefficients of $u(x, y)$. In what follows, sometimes we shall denote $P_{m, n} u$ by $u_{m, n}, u_{m, m}$ by $u_{m}$ and $P_{m, m}$ by $P_{m}$. In connection with operator $P_{m}$, we then have the following theorem.

Theorem 5.2. For function $u(x, y)$ satisfying the conditions of Theorem 5.1, it holds that

$$
\begin{gathered}
\left\|\left(I-P_{m}\right) u\right\|_{T}^{2} \leq \frac{1}{(m+1)^{2}}\left[\left\|\left(I-P_{m-1}\right) u_{x}\right\|_{T}^{2}+\left\|\left(I-P_{m-1}\right) u_{y}\right\|_{T}^{2}\right] \\
(m \geq 1),
\end{gathered}
$$

where $I$ is an identical operator. 
Proof. By the definition of $P_{m}$, we have:

$$
\left(I-P_{m}\right) u \sim \sum_{k \text { or } s \geq m+1}^{\infty} e_{k, s} A_{k, s} T_{k}(x) T_{s}(y) .
$$

Then by Parseval's equality we have:

$$
\frac{1}{\pi^{2}}\left\|\left(I-P_{m}\right) u\right\|_{T}^{2}=\left(\sum_{k=0}^{m} \sum_{s=m+1}^{\infty}+\sum_{s=0}^{\infty} \sum_{k=m+1}^{\infty}\right) A_{k, s}^{2} .
$$

Now let $A_{k, s}^{\prime}$ and $A_{k, s}^{\prime \prime}$ be respectively the Chebyshev coefficients of $u_{x}(x, y)$ and $u_{y}(x, y)$. Then using formula (5.11), we have:

$$
\begin{aligned}
& \frac{1}{\pi^{2}}\left\|\left(I-P_{m}\right) u\right\|_{T}^{2}=\sum_{k=0}^{m} \sum_{s=m+1}^{\infty}\left(\frac{A_{k, s-1}^{\prime \prime}-A_{k, s+1}^{\prime \prime}}{2 s}\right)^{2} \\
& \quad+\sum_{s=0}^{\infty} \sum_{k=m+1}^{\infty}\left(\frac{A_{k-1, s}^{\prime}-A_{k+1, s}^{\prime}}{2 k}\right)^{2} \\
& \quad \leq \frac{1}{2(m+1)^{2}}\left[\sum_{k=0}^{m} \sum_{s=m+1}^{\infty}\left(A_{k, s-1}^{\prime \prime 2}+A_{k, s+1}^{\prime \prime 2}\right)\right. \\
& \left.\quad+\sum_{s=0}^{\infty} \sum_{k=m+1}^{\infty}\left(A_{k-1, s}^{\prime 2}+A_{k+1, s}^{\prime 2}\right)\right] .
\end{aligned}
$$

Applying Parseval's equality to $\left(I-P_{m-1}\right) u_{y}(x, y)$ and $\left(I-P_{m-1}\right) u_{x}(x, y)$, we thus have:

$$
\begin{aligned}
& \frac{1}{\pi^{2}}\left\|\left(I-P_{m}\right) u\right\|_{T}^{2} \leq \frac{1}{\pi^{2}(m+1)^{2}}\left[\left\|\left(I-P_{m-1}\right) u_{y}\right\|_{T}^{2}\right. \\
& \left.\quad+\left\|\left(I-P_{m-1}\right) u_{x}\right\|_{T}^{2}\right],
\end{aligned}
$$

from which (5.15) readily follows. This completes the proof. Q.E.D.

Theorem 5.3. Suppose that $u(x, y)$ is continuous and has piecewise continuous first derivatives in $\bar{S}$. Then it holds that

$$
\left\{\begin{array}{l}
\left\|\frac{\partial}{\partial x}\left(u-u_{m}\right)\right\|_{T}^{2} \leq \frac{m+2}{2}\left\|\left(I-P_{m-1}\right) u_{x}\right\|_{T}^{2} \\
\left\|\frac{\partial}{\partial y}\left(u-u_{m}\right)\right\|_{T}^{2} \leq \frac{m+2}{2}\left\|\left(I-P_{m-1}\right) u_{y}\right\|_{T}^{2} \quad(m \geq 1) .
\end{array}\right.
$$

Proof. By the assumption, the Chebyshev series of $u(x, y)$ converges to $u(x, y)$ itself in $\bar{S}$ (For example, see Hobson [8]). Hence we have

$$
u-u_{m}=\left(I-P_{m}\right) u=\sum_{k \text { or }}^{\infty} e_{s \geq m+1} e_{k, s} A_{k, s} T_{k}(x) T_{s}(y)
$$


Now, let the Chebyshev series of $\partial\left(u-u_{m}\right) / \partial x$ be

$$
\frac{\partial}{\partial x}\left(u-u_{m}\right) \sim \sum_{k, s=0}^{\infty} e_{k, s} \bar{A}_{k, s} T_{k}(x) T_{s}(y) .
$$

Suppose $m$ is even and positive. Then by (5.12) we have:

$(5.22)$

$$
\left\{\begin{array}{l}
\text { for } \quad k, s \leq m, \\
\bar{A}_{0, s}=\sqrt{2}\left[(m+1) A_{m+1, s}+(m+3) A_{m+3, s}+\cdots\right]=\frac{1}{\sqrt{2}} A_{m, s}^{\prime}, \\
\bar{A}_{k, s}=2\left[(m+1) A_{m+1, s}+(m+3) A_{m+3, s}+\cdots\right]=A_{m, s}^{\prime} \\
\quad(k=2,4, \cdots, m), \\
\bar{A}_{k, s}=2\left[(m+2) A_{m+2, s}+(m+4) A_{m+4, s}+\cdots\right]=A_{m+1, s}^{\prime} \\
\quad(k=1,3, \cdots, m-1), \\
\text { for } \quad k \geq m+1 \quad \text { or } \quad s \geq m+1, \quad \\
\bar{A}_{k, s}=2\left[(k+1) A_{k+1, s}+(k+3) A_{k+3, s}+\cdots\right]=A_{k, s}^{\prime} .
\end{array}\right.
$$

Hence from (5.21) we have:

$$
\begin{aligned}
\frac{\partial}{\partial x}( & \left.u-u_{m}\right) \sim \frac{1}{\sqrt{2}} A_{m, 0}^{\prime}+\sqrt{2}\left[\frac{1}{\sqrt{2}} \sum_{s=1}^{m} A_{s, m}^{\prime} T_{s}(y)+\sum_{s=m+1}^{\infty} A_{0, s}^{\prime} T_{s}(y)\right. \\
& +A_{m, 0}^{\prime}\left\{T_{2}(x)+T_{4}(x)+\cdots+T_{m}(x)\right\} \\
& \left.+A_{m+1,0}^{\prime}\left\{T_{1}(x)+T_{3}(x)+\cdots+T_{m-1}(x)\right\}+\sum_{k=m+1}^{\infty} A_{k, 0}^{\prime} T_{k}(x)\right] \\
& +2\left[\sum _ { s = 1 } ^ { m } \left\{A_{m, s}^{\prime}\left(T_{2}(x)+T_{4}(x)+\cdots+T_{m}(x)\right) T_{s}(y)\right.\right. \\
& +A_{m+1, s}^{\prime}\left(T_{1}(x)+T_{3}(x)+\cdots+T_{m-1}(x)\right) T_{s}(y) \\
& \left.\left.+\sum_{k=m+1}^{\infty} A_{k, s}^{\prime} T_{k}(x) T_{s}(y)\right\}+\sum_{s=m+1}^{\infty} \sum_{k=1}^{\infty} A_{k, s}^{\prime} T_{k}(x) T_{s}(y)\right] .
\end{aligned}
$$

Then by Parseval's equality, we have

$$
\begin{aligned}
& \frac{1}{\pi^{2}}\left\|\frac{\partial}{\partial x}\left(u-u_{m}\right)\right\|_{T}^{2}=\frac{m+1}{2} \sum_{s=0}^{m} A_{m, s}^{\prime 2}+\frac{m}{2} \sum_{s=0}^{m} A_{m+1, s}^{\prime 2} \\
& \quad+\sum_{s=0}^{m} \sum_{k=m+1}^{\infty} A_{k, s}^{\prime 2}+\sum_{s=m+1}^{\infty} \sum_{k=0}^{\infty} A_{k, s}^{\prime 2} . \\
& \quad=\frac{m+1}{2} \sum_{s=0}^{m} A_{m, s}^{\prime 2}+\frac{m+2}{2} \sum_{s=0}^{m} A_{m+1, s}^{\prime 2} \\
& \quad+\sum_{s=0}^{m} \sum_{k=m+2}^{\infty} A_{k, s}^{\prime 2}+\sum_{s=m+1}^{\infty} \sum_{k=0}^{\infty} A_{k, s}^{\prime 2} .
\end{aligned}
$$


In a similar way, it is seen that equality (5.23) is valid also when $m$ is odd. Then by Parseval's equality, for any positive integer $m$, we have

$$
\frac{1}{\pi^{2}}\left\|\frac{\partial}{\partial x}\left(u-u_{m}\right)\right\|_{T}^{2} \leq \frac{m+2}{2} \cdot \frac{1}{\pi^{2}}\left\|\left(I-P_{m-1}\right) u_{x}\right\|_{T}^{2},
$$

from which the first inequality of (5.19) readily follows. The second inequality of (5.19) can be derived from the first one by interchanging the variable $x$ with $y$.

Q.E.D.

Theorem 5.4. Suppose that $u(x, y)$ is continuously differentiable and has piecewise continuous second derivatives in $\bar{S}$. Then there is a polynomial $p_{m}(x, y)$ of degree $m$ such that

$$
\left\|u(x, y)-p_{m}(x, y)\right\|_{1, L^{2}(s)}^{2} \leq \widehat{C}_{m}\left(\sum_{i, j}\left\|\left(I-P_{m-2}\right) \partial_{t,}^{2} u\right\|_{T}^{2}\right)
$$

where $\widehat{C}_{m}$ is a number dependent on $m$ such that

$$
\widehat{C}_{m}=O(1 / m) \text { as } m \rightarrow \infty .
$$

Proof. By Theorem 5.2, we have

$$
\begin{aligned}
\left\|\left(I-P_{m}\right) u\right\|_{T}^{2} & \leq \frac{1}{(m+1)^{2}}\left[\left\|\left(I-P_{m-1}\right) u_{x}\right\|_{T}^{2}+\left\|\left(I-P_{m-1}\right) u_{y}\right\|_{T}^{2}\right] \\
& \leq \frac{1}{m^{2}(m+1)^{2}} \cdot \sum_{i, j}\left\|\left(I-P_{m-2}\right) \partial_{i, j}^{2} u\right\|_{T}^{2} .
\end{aligned}
$$

In addition, by Theorem 5.3 and Theorem 5.2, we have:

$$
\left\{\begin{array}{l}
\left\|\frac{\partial}{\partial x}\left(u-u_{m}\right)\right\|_{T}^{2} \leq \frac{m+2}{2}\left\|\left(I-P_{m-1}\right) u_{x}\right\|_{T}^{2} \\
\leq \frac{m+2}{2 m^{2}}\left[\left\|\left(I-P_{m-2}\right) u_{x x}\right\|_{T}^{2}+\left\|\left(I-P_{m-2}\right) u_{x y}\right\|_{T}^{2}\right], \\
\left\|\frac{\partial}{\partial y}\left(u-u_{m}\right)\right\|_{T}^{2} \\
\leq \frac{m+2}{2 m^{2}}\left[\left\|\left(I-P_{m-2}\right) u_{y x}\right\|_{T}^{2}+\left\|\left(I-P_{m-2}\right) u_{y y}\right\|_{T}^{2}\right] .
\end{array}\right.
$$

Hence by the addition of (5.27) and (5.28) we have

$$
\left\|u-u_{m}\right\|_{1, L^{2}(s)}^{2} \leq\left[\frac{1}{m^{2}(m+1)^{2}}+\frac{m+2}{2 m^{2}}\right] \sum_{i, j}\left\|\left(I-P_{m-2}\right) \partial_{i j}^{2} u\right\|_{T}^{2},
$$


which shows that the theorem is valid for $p_{m}(x, y)=u_{m}(x, y)$. This completes the proof.

Q.E. D.

\section{§6. Approximation of a Function Vanishing on the Boundary}

Let $R^{2}$ be $R^{2}=\{(x, y):-\infty<x, y<\infty\}$ and consider a function $K(x, y)$ such that

$$
K(x, y)=\left\{\begin{array}{ccc}
C \exp \left[-1 /\left(1-x^{2}-y^{2}\right)\right] & \text { if } & x^{2}+y^{2}<1 \\
0 & \text { if } & x^{2}+y^{2} \geq 1
\end{array}\right.
$$

where $C$ is a constant such that

$$
\iint_{R^{2}} K(x, y) d x d y=1
$$

In terms of $K(x, y)$, let us define function $K_{\delta}(x, y)$ for arbitrary positive number $\delta$ in a following way:

$$
K_{\delta}(x, y)=\delta^{-2} K(x / \delta, y / \delta) .
$$

For any $u(x, y) \in L^{2}\left(R^{2}\right)$, put

$$
\tilde{u}_{\delta}(x, y)=\iint_{R^{2}} K_{\delta}(x-t, y-s) u(t, s) d t d s,
$$

then it is readily seen that $\tilde{u}_{\delta}(x, y) \in \stackrel{\circ}{C}^{\infty}\left(R^{2}\right)$ and the support of $\tilde{u}_{\delta}$ lies in the $\delta$-neighborhood of the support of $u(x, y)$.

Now, for the given domain $D$, let us assume the condition $(B)$ :

(i) $\bar{D}$ lies in the interior of the square $S$ specified in $\S 5$,

$$
\iint_{D-D_{\delta}} \rho^{-\kappa}(x, y) d x d y=O\left(\delta^{1-\kappa}\right)
$$

for any positive number $\kappa<1$ as $\delta \rightarrow 0$, where

$$
D_{\delta}=\{p \in D: \operatorname{dist}(p, \partial D)>\delta\}
$$

and $\rho(x, y)=\operatorname{dist}((x, y), \partial D)$. As easily verified, the equality in (ii) holds when the boundary of $D$ consists of a finite number of twice continuously differentiable arcs.

Let us note that the equality in (ii) implies

$$
\operatorname{mes}\left(D-D_{\delta}\right)=O(\delta) \quad(\delta \rightarrow 0),
$$


where $\operatorname{mes}\left(D-D_{\delta}\right)$ is the measure of the set $D-D_{\delta}$. In fact, let $(x, y)$ be an arbitrary point belonging to $D-D_{\delta}$, then

$$
\rho(x, y) \leq \delta
$$

therefore

$$
\rho^{-\kappa}(x, y) \geq \delta^{-\kappa}
$$

Then by (ii), we have

$$
\delta^{-\kappa} \iint_{D-D_{\delta}} d x d y \leq \iint_{D-D_{\delta}} \rho^{-\kappa}(x, y) d x d y=O\left(\delta^{1-\kappa}\right),
$$

from which we readily get (6.4).

Now, for the domain $D$ satisfying condition $(B)$, let us prove the following theorem.

Theorem 6.1. Suppose that, for the function $u(x, y) \in \dot{C}^{2}(D)$, there is a positive constant $\rho_{0}$ such that inequality $\rho(x, y)=\operatorname{dist}((x, y)$, $\partial D) \leq \rho_{0}$ implies

$$
|u(x, y)| \leq \widetilde{C}_{1} \rho^{\kappa_{1}}(x, y) \quad\left(\kappa_{1}>\frac{1}{2}\right)
$$

for some positive constants $\widetilde{C}_{1}$ and $\kappa_{1}$, and the inequality $0<\rho<\rho_{0}$ implies

$$
\sum_{i=1}^{2}\left|\partial_{2} u(x, y)\right|^{2} \leq \widetilde{C}_{2} \rho^{-\kappa_{2}} \quad\left(\kappa_{2}<1\right)
$$

on $\overline{D_{\rho}}$ and

$$
\iint_{D_{\rho} \sum_{i, \rho}}\left|\partial_{\imath,}^{2} u(x, y)\right|^{2} d x d y \leq \widetilde{C}_{3} \rho^{-\kappa_{3}} \quad\left(\kappa_{3}<2\right)
$$

for some positive contants $\widetilde{C}_{2}, \widetilde{C}_{3}, \kappa_{2}$ and $\kappa_{3}$.

Then there is a polynomial $p_{m}(x, y)$ of degree $m$ such that

$$
\left\|u(x, y)-\omega(x, y) p_{m}(x, y)\right\|_{1, L^{2}(D)}^{2} \leq \widetilde{C_{4}} \frac{1}{m^{\alpha}} \quad(m \rightarrow \infty)
$$

for some positive constants $\alpha$ and $\widetilde{C_{4}}$, where $\omega(x, y)$ is a function satisfying the conditions stated in $\S 2$.

Proof. Let $\delta$ be an arbitrary number such that $0<5 \delta<\rho_{0}$, and consider the function $u_{\delta}(x, y)$ such that 


$$
u_{\delta}(x, y)=\left\{\begin{array}{cl}
u(x, y) & (x, y) \in \bar{D}_{2 \delta} \\
0 & (x, y) \in R^{2}-\bar{D}_{2 \delta}
\end{array}\right.
$$

Put

$$
\tilde{u}_{\delta}(x, y)=\iint_{R^{2}} K_{\delta}(x-t, y-s) u_{\delta}(t, s) d t d s,
$$

then $\tilde{u}_{\delta}(x, y) \in \stackrel{\circ}{C}^{\infty}\left(R^{2}\right)$ and $\operatorname{supp}\left(\tilde{u}_{\delta}\right) \subset \overline{D_{\delta}}$. For function $\tilde{u}_{\delta}(x, y)$, we shall prove that there are three positive constants $C_{1}, C_{2}, \kappa_{4}$ independent of $\delta$ such that

$$
\left\{\begin{array}{l}
\left\|u(x, y)-\tilde{u}_{\delta}(x, y)\right\|_{1, L^{2}(D)}^{2} \leq C_{1} \delta^{K_{4}} . \\
\max _{\bar{D}_{\delta}}\left|\partial_{i j}^{2} \tilde{u}_{\delta}(x, y)\right| \leq C_{2} \delta^{-2} \quad(i, j=1,2) .
\end{array}\right.
$$

First, let us consider the quantity

$$
\begin{aligned}
\left\|u(x, y)-\tilde{u}_{\delta}(x, y)\right\|_{L^{2}(D)}^{2}= & \left\|u(x, y)-\tilde{u}_{5}(x, y)\right\|_{L^{2}\left(D_{3 \delta}\right)}^{2} \\
& +\left\|u(x, y)-\tilde{u}_{\delta}(x, y)\right\|_{L^{2}\left(D-D_{3 \delta}\right)}^{2} .
\end{aligned}
$$

For the first quantity in the right-hand side, by (b), we have:

$$
\begin{aligned}
& \left.\left\|u(x, y)-\tilde{u}_{\delta}(x, y)\right\|_{L^{2}\left(D_{3 \delta}\right)}^{2}\right) \\
& \quad=\iint_{D_{3 \delta}}\left[\iint_{t^{2}+s^{2} \leq \delta^{2}} K_{\delta}(t, s)\{u(x, y)-u(x-t, y-s)\} d t d s\right]^{2} d x d y \\
& \quad=\iint_{D_{3 \delta}}\left[\int \int _ { t ^ { 2 } + s ^ { 2 } \leq \delta ^ { 2 } } K _ { \delta } ( t , s ) \left\{\int _ { 0 } ^ { 1 } \left(u_{x}(x-\theta t, y-\theta s) t\right.\right.\right. \\
& \left.\left.\left.\quad+u_{y}(x-\theta t, y-\theta s) s\right) d \theta\right\} d t d s\right]^{2} d x d y \\
& \quad \leq \operatorname{mes}\left(D_{3 \delta}\right) \cdot\left[\sqrt{\widetilde{C}_{2}} \cdot(2 \delta)^{-\kappa_{2} / 2} \delta\right]^{2} \\
& \quad=\operatorname{const.} \times \delta^{2-\kappa_{2}} .
\end{aligned}
$$

On the other hand, for the second quantity in the right-hand side of (6.9), by (a), we have:

$$
\begin{aligned}
& \left\|u(x, y)-\tilde{u}_{\delta}(x, y)\right\|_{L^{2}\left(D-D_{3 \delta}\right)}^{2} \\
& \quad \leq\left(2 \max _{\rho(x, y) \leq 4 \delta}|u|\right)^{2} \cdot \operatorname{mes}\left(D-D_{3 \delta}\right) \\
& \quad \leq \text { const. } \times \delta^{1+2 \kappa_{1}} .
\end{aligned}
$$

Thus for the quantity of (6.9), we have

$$
\left\|u(x, y)-\tilde{u}_{\delta}(x, y)\right\|_{L^{2}(D)}^{2} \leq \text { const. } \times \delta .
$$


Next, let us consider the quantity

$$
\begin{aligned}
& \left\|\partial_{x} u(x, y)-\partial_{x} \tilde{u}_{\delta}(x, y)\right\|_{L^{2}(D)}^{2} \\
& =\left\|\partial_{x} u(x, y)-\partial_{x} \tilde{u}_{\delta}(x, y)\right\|_{L^{2}\left(D_{3 \delta}\right)}^{2}+\left\|\partial_{x} u(x, y)-\partial_{x} \tilde{u}_{\delta}(x, y)\right\|_{L^{2}\left(D-D_{3 \delta}\right) \cdot}^{2}
\end{aligned}
$$

For the first quantity in the right-hand side, by (c), we have:

$$
\begin{aligned}
& \left\|\partial_{x} u(x, y)-\partial_{x} \tilde{u}_{\delta}(x, y)\right\|_{L^{2}\left(D_{3 \delta}\right)}^{2} \\
& =\iint_{D_{3 \delta}}\left[\iint_{t^{2}+s^{2} \leq \delta^{2}} K_{\delta}(t, s)\left\{u_{x}(x, y)-u_{x}(x-t, y-s)\right\} d t d s\right]^{2} d x d y \\
& =\iint_{D_{3 \delta}}\left[\int \int _ { t ^ { 2 } + s ^ { 2 } \leq \delta ^ { 2 } } K _ { \delta } ( t , s ) \left\{\int _ { 0 } ^ { 1 } \left(u_{x x}(x-\theta t, y-\theta s) t\right.\right.\right. \\
& \left.\left.\left.+u_{x y}(x-\theta t, y-\theta s) s\right) d \theta\right\} d t d s\right]^{2} d x d y \\
& =\iint_{D_{3 \delta}}\left[\int _ { 0 } ^ { 1 } \left\{\int \int _ { t ^ { 2 } + s ^ { 2 } \leq \delta ^ { 2 } } K _ { \delta } ( t , s ) \left(u_{x x}(x-\theta t, y-\theta s) t\right.\right.\right. \\
& \left.\left.\left.+u_{x y}(x-\theta t, y-\theta s) s\right) d t d s\right\} d \theta\right]^{2} d x d y \\
& \leq \iint_{D_{3 \delta}}\left[\int _ { 0 } ^ { 1 } \left\{\int \int _ { t ^ { 2 } + s ^ { 2 } \leq \delta ^ { 2 } } K _ { \delta } ( t , s ) \left(u_{x x}(x-\theta t, y-\theta s) t\right.\right.\right. \\
& \left.\left.\left.+u_{x y}(x-\theta t, y-\theta s) s\right) d t d s\right\}^{2} d \theta\right] d x d y \leq \text { const. } \times \delta^{-4} \\
& \times \iint_{D_{3 \delta}}\left[\int_{0}^{1} \iiint_{t^{2}+s^{2} \leq \delta^{2}} \sqrt{u_{x x}^{2}(x-\theta t, y-\theta s)+u_{x y}^{2}(x-\theta t, y-\theta s)}\right. \\
& \left.\left.\times \sqrt{\bar{t}^{2}+s^{2}} d t d s\right\}^{2} d \theta\right] d x d y \\
& \leq \text { const. } \times \int_{0}^{1}\left[\int \int _ { D _ { 3 \delta } } \left\{\int \int _ { t ^ { 2 } + s ^ { 2 } \leq \delta ^ { 2 } } \left(u_{x x}^{2}(x-\theta t, y-\theta s)\right.\right.\right. \\
& \left.\left.\left.+u_{x y}^{2}(x-\theta t, y-\theta s)\right) d t d s\right\} d x d y\right] d \theta \\
& \leq \text { const. } \times \int_{0}^{1}\left[\int \int _ { t ^ { 2 } + s ^ { 2 } \leq \delta ^ { 2 } } \left\{\int \int _ { D _ { 2 \delta } } \left(u_{x x}^{2}(x, y)\right.\right.\right. \\
& \left.\left.\left.+u_{x y}^{2}(x, y)\right) d x d y\right\} d t d s\right] d \theta \\
& \leq \text { const. } \times \delta^{2-\kappa_{3}} \text {. }
\end{aligned}
$$

To estimate the second quantity in the right-hand side of (6.13), let us consider 


$$
\partial_{x} \tilde{u}_{\delta}(x, y)=\iint_{\substack{(x-t) 2 \\+(y-s)^{2} \leq \delta^{2}}} \partial_{x} K_{\delta}(x-t, y-s) u_{\delta}(t, s) d t d s
$$

Since

$$
\partial_{x} K_{\delta}(x-t, y-s)=\delta^{-3} K_{x}^{\prime}[(x-t) / \delta,(y-s) / \delta]
$$

we then have

$$
\left|\partial_{x} \tilde{u}_{\delta}(x, y)\right| \leq \text { const. } \times \delta^{-3} \times \iint_{\substack{(x-t) 2 \\(y-s)^{2} \leq \delta^{2}}} u_{\delta}(t, s) d t d s .
$$

Then by (a), for $(x, y) \in D-D_{3 \delta}$, we have

$$
\begin{aligned}
\left|\partial_{x} \tilde{u}_{\delta}(x, y)\right| & \leq \text { const. } \times \delta^{-3} \times \widetilde{C}_{1}(4 \delta)^{\kappa_{1}} \times \pi \delta^{2} \\
& \leq \text { const. } \times \delta^{\kappa_{1}-1}
\end{aligned}
$$

and hence

$$
\begin{aligned}
\left\|\partial_{x} u_{\delta}(x, y)\right\|_{L^{2}\left(D-D_{3 \delta}\right)}^{2} & \leq \text { const. } \times \delta^{2\left(\kappa_{1}-1\right)} \times \operatorname{mes}\left(D-D_{3 \delta}\right) \\
& \leq \text { const. } \times \delta^{2 \kappa_{1}-1} .
\end{aligned}
$$

Since (b) implies

$$
\sum_{i=1}^{2}\left|\partial_{i} u(x, y)\right|^{2} \leq \widetilde{C}_{2} \rho^{-\kappa_{2}}(x, y)
$$

by (ii), we have

$$
\left\|\partial_{x} u(x, y)\right\|_{L^{2}\left(D-D_{3 \delta}\right)}^{2} \leq \text { const. } \times \delta^{1-\kappa_{2}} .
$$

Then, since

$$
\begin{aligned}
\| \partial_{x} u(x, y) & -\partial_{x} \tilde{u}_{\delta}(x, y) \|_{L^{2}\left(D-D_{3 \delta}\right)}^{2} \\
& \leq 2\left[\left\|\partial_{x} u(x, y)\right\|_{L^{2}\left(D-D_{3 \delta}\right)}^{2}+\left\|\partial_{x} \tilde{u}_{\delta}(x, y)\right\|_{L^{2}\left(D-D_{3 \delta}\right.}^{2}\right],
\end{aligned}
$$

by (6.18) and (6.19), we have

$$
\left\|\partial_{x} u(x, y)-\partial_{x} \tilde{U}_{\delta}(x, y)\right\|_{L^{2}\left(D-D_{3 \delta}\right)}^{2} \leq \text { const. } \times \delta^{\kappa_{5}}
$$

for some $\kappa_{5}>0$. From (6.14) and (6.20), it then follows that

$$
\left\|\partial_{x} u(x, y)-\partial_{x} \tilde{u}_{\delta}(x, y)\right\|_{L^{2}(D)}^{2} \leq \text { const. } \times \delta^{\kappa_{6}}
$$

for some $\kappa_{6}>0$. The analogous estimate can be obtained similarly for

$$
\left\|\partial_{y} u(x, y)-\partial_{y} \tilde{u}_{\delta}(x, y)\right\|_{L^{2}(D)}^{2} .
$$

Then combining these estimates with (6.12), we have the first inequality of (6.8). The second inequality of (6.8) can be proved easi- 
ly from the equations:

$$
\partial_{i j}^{2} \tilde{u}_{\delta}(x, y)=\iint_{R^{2}} \partial_{i j}^{2} K_{\delta}(x-t, y-s) u_{\delta}(t, s) d t d s \quad(i, j=1,2) .
$$

Now we shall prove the existence of a polynomial $p_{m}(x, y)$ satisfying (6.5).

Consider the function $v_{\delta}(x, y)$ defined by

$$
v_{\delta}(x, y)=\left\{\begin{array}{cl}
\tilde{u}_{\delta}(x, y) / \omega(x, y) & \text { in } D \\
0 & \text { in } R^{2}-D
\end{array}\right.
$$

Since the support of $\tilde{u}_{\delta}(x, y)$ lies in $\bar{D}_{\delta}$, the support of $v_{\delta}(x, y)$ lies also in $\bar{D}_{\delta}$. Moreover by the condition (II) for $\omega(x, y), v_{\delta}(x, y)$ is continuously differentiable in $\bar{S}$. Hence, by Theorem 5.4, there is a polynomial $p_{m}(x, y)$ of degree $m$ such that

$$
\left\|v_{\delta}(x, y)-p_{m}(x, y)\right\|_{1, L^{2}(D)}^{2} \leq C_{3}\left(\sum_{i, j}\left\|\partial_{i j}^{2} v_{\delta}\right\|_{T}^{2}\right) \frac{1}{m} .
$$

Let us estimate the quantity $\left\|\partial_{i j}^{2} v_{\delta}\right\|_{T}^{2}$. Since $\operatorname{supp}\left(v_{\delta}\right) \subset \overline{D_{\delta}} \subset D$ and $\bar{D}$ lies in the interior of $S$, we have

$$
\begin{aligned}
\left\|\partial_{i j}^{2} v_{\delta}\right\|_{T}^{2} & =\iint_{s} \frac{\left(\partial_{i j}^{2} v_{\delta}\right)^{2}}{\sqrt{1-x^{2}} \sqrt{1-y^{2}}} d x d y \\
& =\iint_{D_{\delta}} \frac{\left(\partial_{i j}^{2} v_{\delta}\right)^{2}}{\sqrt{1-x^{2}} \sqrt{1-y^{2}}} d x d y \\
& \leq \text { const. } \times \iint_{D_{\delta}}\left(\partial_{\imath}^{2} v_{\delta}\right)^{2} d x d y
\end{aligned}
$$

However by (6.23), on $D_{\delta}$, we have:

$$
\begin{aligned}
\partial_{i j}^{2} v_{\delta}= & {\left[\omega^{2} \partial_{i j}^{2} \tilde{u}_{\delta}-\omega \tilde{u}_{\delta} \cdot \partial_{i j}^{2} \omega-\omega\left(\partial_{i} \tilde{u}_{\delta} \cdot \partial_{j} \omega+\partial_{j} \tilde{u}_{\delta} \cdot \partial_{i} \omega\right)\right.} \\
& \left.+2 \tilde{u}_{\delta} \cdot \partial_{i} \omega \cdot \partial_{j} \omega\right] / \omega^{3} .
\end{aligned}
$$

Let us suppose $0<\delta \leq \bar{\delta} \leq 1$ where $\bar{\delta}$ is a number stated in condition (III) for $\omega(x, y)$. Then, by the assumptions on $\omega(x, y)$, we have

$$
\left\{\begin{array}{l}
|\omega(x, y)|,\left|\partial_{i} \omega(x, y)\right| \leq \text { const. } \quad(\mathrm{i}=1,2), \\
\omega(x, y) \geq \min \left[\min _{D_{\bar{\delta}}} \omega(x, y), \bar{C}_{2}\right] \cdot \delta^{\lambda_{2}}, \\
\mid \partial_{i j}^{2} \omega(x, y) \leq \max \left[\max _{D_{\bar{\delta}}}\left|\partial_{i j}^{2} \omega(x, y)\right|, \bar{C}_{1}\right] \cdot \delta^{-\lambda_{1}}
\end{array}\right.
$$


on $\bar{D}_{\delta}$. On the other hand, by (6.7) and the second of (6.8), we have

$$
\begin{cases}\left|\tilde{u}_{\delta}(x, y)\right| \leq \text { const., } & \\ \left|\partial_{i} \tilde{u}_{\delta}(x, y)\right| \leq \text { const. } \times \delta^{-1} & (i=1,2), \\ \left|\partial_{i j}^{2} \tilde{u}_{\delta}(x, y)\right| \leq C_{2} \delta^{-2} & (i, j=1,2)\end{cases}
$$

on $\bar{D}_{\delta}$. Hence we see that

$$
\begin{aligned}
\partial_{i j}^{2} v_{\delta} & =O\left(\delta^{-3 \lambda_{2}}\right)\left[O\left(\delta^{-2}\right)+O\left(\delta^{-\lambda_{1}}\right)+O\left(\delta^{-1}\right)+O(1)\right] \\
& =O\left(\delta^{-3 \lambda_{2}}\right)\left[O\left(\delta^{-2}\right)+O\left(\delta^{-\lambda_{1}}\right)\right] \quad(\delta \rightarrow 0) .
\end{aligned}
$$

Then from (6.25), we have

$$
\left\|\partial_{i j}^{2} v_{\delta}\right\|_{T}^{2} \leq \text { const. } \times \delta^{-6 \lambda_{2}}\left(\delta^{-4}+\delta^{-2 \lambda_{1}}\right)
$$

for sufficiently small $\delta$, because

$$
\left\{\begin{array}{lll}
\delta^{-2-\lambda_{1}} \leq \delta^{-4} & \text { for } & \lambda_{1} \leq 2, \\
\delta^{-2-\lambda_{1}} \leq \delta^{-2 \lambda_{1}} & \text { for } & \lambda_{1} \geq 2 .
\end{array}\right.
$$

Now put $\delta=m^{-\alpha_{1}}$, then from (6.24) and (6.30) follows

$$
\begin{aligned}
& \left\|v_{\delta}(x, y)-p_{m}(x, y)\right\|_{1, L^{2}(D)}^{2} \\
& \quad \leq \text { const. } \times m^{6 \lambda_{2} \alpha_{1}}\left(m^{4 \alpha_{1}}+m^{2 \lambda_{1} \alpha_{1}}\right) m^{-1} .
\end{aligned}
$$

In order that

$$
m^{6 \lambda_{2} \alpha_{1}}\left(m^{4 \alpha_{1}}+m^{2 \lambda_{1} \alpha_{1}}\right) m^{-1} \leq \text { const. } \times m^{-\alpha_{2}}
$$

for a positive number $\alpha_{2}<1$, it is sufficient that

$$
\alpha_{1} \leq \frac{1-\alpha_{2}}{6 \lambda_{2}+4+2 \lambda_{1}} .
$$

Thus we see that if $m$ is sufficiently large, then for a positive number $\alpha_{1}$ satisfying the above inequality, we have

$$
\left\|v_{m}(x, y)-p_{m}(x, y)\right\|_{1, L^{2}(D)}^{2} \leq C_{4} m^{-\alpha_{2}}
$$

for some positive constant $C_{4}$, where $v_{m}(x, y)=v_{\delta}(x, y)$ for $\delta=m^{-\alpha_{1}}$. On the other hand, from the first of (6.8), we have

$$
\left\|u(x, y)-\tilde{u}_{u}(x, y)\right\|_{1, L^{2}(D)}^{2} \leq C_{1} m^{-\alpha_{3}}
$$

for sufficiently large $m$, where $\tilde{u}_{m}(x, y)=\tilde{u}_{\delta}(x, y)$ for $\delta=m^{-\alpha_{1}}$ and $\alpha_{3}=$ $\kappa_{4} \alpha_{1}>0$. Put $\alpha=\min \left(\alpha_{2}, \alpha_{3}\right)>0$, then inequalities (6.34) and (6.35) 
imply:

$$
\begin{aligned}
& \left\|u(x, y)-\tilde{u}_{m}(x, y)\right\|_{1, L^{2}(D)}^{2} \leq C_{1} m^{-\alpha} \\
& \left\|v_{m}(x, y)-p_{m}(x, y)\right\|_{1, L^{2}(D)}^{2} \leq C_{1} m^{-\alpha}
\end{aligned}
$$

for sufficiently large $m$.

Now, since $\omega(x, y)$ and $\partial_{i} \omega(x, y)(i=1,2)$ are all bounded in $\bar{D}$, for sufficiently large $m$, from (6.37) we have:

$$
\begin{aligned}
& \left\|\tilde{u}_{m}(x, y)-\omega(x, y) p_{m}(x, y)\right\|_{L^{2}(D)}^{2} \\
& \quad \leq \text { const. } \times\left\|v_{m}(x, y)-p_{m}(x, y)\right\|_{L^{2}(D)}^{2} \\
& \quad \leq \text { const. } \times m^{-\alpha},
\end{aligned}
$$

and

$$
\begin{aligned}
& \left\|\partial_{i} \tilde{u}_{m}(x, y)-\partial_{i}\left[\omega(x, y) p_{m}(x, y)\right]\right\|_{L^{2}(D)}^{2} \\
& \quad=\left\|\partial_{i}\left[\omega(x, y) v_{m}(x, y)\right]-\partial_{i}\left[\omega(x, p) p_{m}(x, y)\right]\right\|_{L^{2}(D)}^{2} \\
& \quad \leq \text { const. } \times\left\|v_{m}(x, y)-p_{m}(x, y)\right\|_{L^{2}(D)}^{2} \\
& \quad+\text { const. } \times\left\|\partial_{i} v_{m}(x, y)-\partial_{i} p_{m}(x, y)\right\|_{L^{2}(D)}^{2} \\
& \quad \leq \text { const. } \times m^{-\alpha} \quad(i=1,2) .
\end{aligned}
$$

Hence we have

$$
\left\|\tilde{u}_{m}(x, y)-\omega(x, y) p_{m}(x, y)\right\|_{1, L^{2}(D)}^{2} \leq C_{5} m^{-\alpha}
$$

for some positive constant $C_{5}$ provided $m$ is sufficiently large. From (6.36) and (6.38), follows inequality (6.5). This completes the proof.

Q. E. D.

\section{§7. The Uniform Convergence of Approximate Solutions}

A bounded domain $D$ with boundary $\Gamma$ is said to satisfy condition (C) if for every point $p \in \bar{D}$ there is a closed line segment $l_{p}$ centered at $p$ and of a fixed length satisfying the following conditions:

(a) $l_{p}$ intersects $\Gamma$ in one point at most;

(b) for some $\delta_{0}, l_{p}(\delta) \equiv l_{p} \cap \bar{D}_{\delta} \neq \phi$ whenever $0<\delta \leq \delta_{0}$;

(c) if $\hat{l}_{p}(\delta) \equiv l_{p} \cap\left(\bar{D}-\bar{D}_{\delta}\right) \neq \phi$ for $0<\delta \leq \delta_{0}$, then $l_{p}(\delta)$ and $\hat{l}_{p}(\delta)$ are both single line segments and 


$$
\text { length }\left[\hat{l}_{p}(\delta)\right] \leq K_{0} \delta
$$

for some positive constant $K_{0}$.

If domain $D$ satisfies condition $(C)$, then we can easily see that for any positive number $\kappa<1$ there is a positive constant $M_{\kappa}$ dependent only on $\kappa$ such that

$$
\int_{l_{\rho} \cap \bar{D}} \rho^{-\kappa}(x, y) d s \leq M_{\kappa}
$$

where $\rho(x, y)=\operatorname{dist}((x, y), \Gamma)$.

In fact, when $\hat{l}_{p}\left(\delta_{0}\right)=\phi$, the assertion is evident. When $\hat{l}_{p}\left(\delta_{0}\right) \neq \phi$, let $s$ be the distance between a pcint $q$ on $\hat{l}_{p}\left(\delta_{0}\right)$ and the end point of $\hat{l}_{p}\left(\delta_{\theta}\right)$ lying ouside $\bar{D}_{\delta_{0}}$. Then by (c), $s \leq K_{0} \rho(s)$, where $\rho(s)=\operatorname{dist}(q, \Gamma)$. Thus it follows that

$$
\int_{\hat{\imath}_{p}\left(\delta_{0}\right)} \rho^{-\kappa}(s) d s \leq K_{0}^{\kappa} \int_{\hat{\imath}_{p}\left(\delta_{0}\right)} s^{-\kappa} d s \leq K_{0}^{\kappa} \frac{\left(L_{0}\right)^{1-\kappa}}{1-\kappa},
$$

where $L_{0}$ is the length of $l_{p}$. This proves our assertion.

Theorem 7.1. Let $D$ be a domain satisfying the conditions $(A)$, $(B)$ and $(C)$, and $\omega(x, y)$ be a function with properties stated in $\S 2$. Let $u(x, y)$ be the solution of the boundary value problem (1.1) satisfying the conditions in Theorem 6.1, then the approximate solution $u_{m}(x, y)$ of the form

$$
u_{m}(x, y)=\omega(x, y) \bar{p}_{m}(x, y)=\omega(x, y) \sum_{k, s=0}^{m} a_{k, s} x^{k} y^{s}
$$

obtained by Ritz-Galerkin's method converges to solution $u(x, y)$ uniformly in $\bar{\nu}$ as $m \rightarrow \infty$, and it holds that

$$
\max _{\bar{D}}\left|u_{m}(x, y)-u(x, y)\right|=O\left(\sqrt{m^{-\alpha} \log m}\right) \quad(m \rightarrow \infty)
$$

for some positive number $\alpha$.

Remark. Inequalities (a) and (b) in Theorem 6.1 are valid for solution $u(x, y)$ if domain $D$ satisfies Pcincaré condition (see [6], [7]). However even if $D$ satisfies Poincare condition, it is not known whether or not inequality (c) in Theorem 6.1 is valid for solution $u(x, y)$. Hence in Theorem 7.1 all these inequalities are assumed to be valid for solution $u(x, y)$. 
To prove Theorem 7.1, we shall use the theorem of Markov (see $[9])$ which reads as follows.

If a polynomial $p(x)$ of degree $m$ satisfies the inequality

$$
|p(x)| \leq M
$$

on the segment of length $h$, then $p^{\prime}(x)$ satisfies

$$
\left|p^{\prime}(x)\right| \leq \frac{2 M}{h} m^{2}
$$

on the same segment.

Proof of Theorem 7.1. In Theorem 6.1, put

$$
\left\{\begin{array}{l}
\hat{u}_{m}(x, y)=\omega(x, y) p_{m}(x, y), \\
\hat{\eta}_{m}(x, y)=u(x, y)-\hat{u}_{m}(x, y),
\end{array}\right.
$$

then by (6.5) we have

$$
\iint_{D}\left[\left(\frac{\partial \hat{\eta}_{m}}{\partial x}\right)^{2}+\left(\frac{\partial \hat{\eta}_{m}}{\partial y}\right)^{2}+\hat{\eta}_{m}^{2}\right] d x d y \leq \text { const. } \times m^{-\alpha} \quad(m \rightarrow \infty)
$$

for some $\alpha>0$. On the other hand, if we put

$$
\eta_{m}(x, y)=u(x, y)-u_{m}(x, y),
$$

then by (4.10) we have

$$
\begin{aligned}
& \iint_{D}\left[a\left(\frac{\partial \eta_{m}}{\partial x}\right)^{2}+b\left(\frac{\partial \eta_{m}}{\partial y}\right)^{2}+c \eta_{m}^{2}\right] d x d y \\
& \quad \leq \iint_{D}\left[a\left(\frac{\partial \widehat{\eta}_{m}}{\partial x}\right)^{2}+b\left(\frac{\partial \widehat{\eta}_{m}}{\partial y}\right)^{2}+c \widehat{\vartheta}_{m}^{2}\right] d x d y .
\end{aligned}
$$

Now put

$$
\begin{aligned}
& M=\max \left[\max _{\bar{D}} a, \max _{\bar{D}} b, \max _{\bar{D}} c\right]>0 \\
& S=\min \left[\min _{\bar{D}} a, \min _{\bar{D}} b\right]>0,
\end{aligned}
$$

then it is evident that

$$
\begin{aligned}
S \cdot I\left(\eta_{m}\right) & =S \iint_{D}\left[\left(\frac{\partial \eta_{m}}{\partial x}\right)^{2}+\left(\frac{\partial \eta_{m}}{\partial y}\right)^{2}\right] d x d y \\
& \leq \iint_{D}\left[a\left(\frac{\partial \eta_{m}}{\partial x}\right)^{2}+b\left(\frac{\partial \eta_{m}}{\partial y}\right)^{2}+c \eta_{m}^{2}\right] d x d y
\end{aligned}
$$

and 


$$
\begin{aligned}
& \iint_{D}\left[a\left(\frac{\partial \hat{\eta}_{m}}{\partial x}\right)^{2}+b\left(\frac{\partial \hat{\eta}_{m}}{\partial y}\right)^{2}+c \hat{\eta}_{m}^{2}\right] d x d y \\
& \quad \leq M \iint_{D}\left[\left(\frac{\partial \hat{\eta}_{m}}{\partial x}\right)^{2}+\left(\frac{\partial \hat{\eta}_{m}}{\partial y}\right)^{2}+\hat{\eta}_{m}^{2}\right] d x d y .
\end{aligned}
$$

Hence from (7.7) and (7.9), we have

$$
J\left(\eta_{m}\right) \leq \text { const. } \times m^{-\alpha} \quad(m \rightarrow \infty)
$$

for some $\alpha>0$.

In order to apply Theorem 4.1 to our function $\eta_{m}(x, y)$, let us check condition (ii) in the same theorem. Put

$$
M_{0}=\max _{\bar{D}}|u(x, y)|, M_{m}=\max _{\bar{D}}\left|\eta_{m}(x, y)\right| .
$$

and let $p$ be an arbitrary point in $\bar{D}$ and $l_{p}$ be the closed line segment corresponding to $p$ in the way stated in the beginning of $\$ 7$. For our solution $u(x, y)$, by (b) in Theorem 6.1 , we have

$$
\begin{aligned}
\left(\frac{d u}{d s}\right)^{2} & \leq\left(\left|\frac{\partial u}{\partial x}\right|+\mid \frac{\partial u}{\partial y}\right)^{2} \\
& \leq 2 \sum_{i=1}^{2}\left|\partial_{i} u\right|^{2} \\
& \leq 2 \widetilde{C}_{2} \rho^{-\kappa_{2}}(x, y) \quad\left(0<\kappa_{2}<1\right)
\end{aligned}
$$

therefore by (7.1) we have

$$
\int_{l_{p} \cap \bar{D}}\left(\frac{d u}{d s}\right)^{2} d s \leq 2 \widetilde{C}^{2} \int_{l_{p} \cap \bar{D}} \rho^{-\kappa_{2}}(x, y) d s \leq K
$$

for some constant $K$ independent of $l_{p}$.

To estimate $d u_{m} / d s$, let us suppose that $m$ is so large that

Put

$$
m^{-3} \leq \delta_{0} / 2
$$

then by (2.2) we have

$$
\delta=m^{-3},
$$

$$
\omega(x, y) \geq \text { const. } \times \delta^{\lambda_{2}} \quad \text { on } \quad l_{p}(\delta)
$$

and hence

$$
\left|\bar{p}_{m}(x, y)\right| \leq \text { const. } \times \delta^{-\lambda_{2}} \cdot \max _{\bar{D}}\left|u_{m}(x, y)\right| \text { on } l_{p}(\delta) \text {. }
$$


Let us denote the quantity in the right-hand side of the above inequality by $R_{m}$. For $l_{p}(\delta)$, by (b) of condition (C), it is clear that

$$
\text { length }\left[l_{p}(\delta)\right] \geq \delta_{0}-\delta \geq \delta_{0} / 2 \text {. }
$$

Now suppose $\hat{l}_{p}(\delta) \neq \phi$. Then by (c) of condition (C), any point $(\tilde{x}, \tilde{y})$ on $\hat{l}_{p}(\delta)$ can be expressed as follows:

$$
\tilde{x}=x_{0}+l_{1} s, \tilde{y}=y_{0}+l_{2} s \quad\left(|s| \leq K_{0} \delta\right)
$$

where $\left(l_{1}, l_{2}\right)$ are direction cosines of $l_{p}$ and $\left(x_{0}, y_{0}\right)$ is a point on $l_{p}(\delta)$. By (7.15), we then have

$$
\bar{p}_{m}(\tilde{x}, \tilde{y})=\bar{p}_{m}\left(x_{0}, y_{0}\right)+\sum_{r=1}^{2 m} \frac{1}{r !}\left[\frac{d^{r}}{d s^{r}} \bar{p}_{m}\left(x_{0}+l_{1} s, y_{0}+l_{2} s\right)\right]_{s=0} \cdot s^{r} .
$$

However, by the theorem of Markov, from (7.14) follows

$$
\begin{aligned}
\left|\frac{d}{d s} \bar{p}_{m}(x, y)\right| & \leq 2 R_{m}(2 m)^{2} / \text { length }\left[l_{p}(\delta)\right] \\
& \leq C_{1}^{\prime} R_{m} m^{2} \text { on } l_{p}(\delta),
\end{aligned}
$$

where $C_{1}^{\prime}=16 / \delta_{0}$.

By the theorem of Markov, from (7.17) it follows successively that

$$
\left|\frac{d^{r}}{d s^{r}} \bar{p}_{m}(x, y)\right| \leq\left(C_{1}^{\prime} m^{2}\right)^{r} \cdot R_{m} \quad \text { on } \quad l_{p}(\delta) \quad(r=2,3, \cdots)
$$

Then from (7.16), we see that

$$
\begin{aligned}
\left|\bar{p}_{m}(\tilde{x}, \tilde{y})\right| & \leq\left[1+\sum_{r=1}^{2 m} \frac{1}{r !}\left(C_{1}^{\prime} m^{2}\right)^{r} \cdot\left(K_{0} \delta\right)^{r}\right] R_{m} \\
& =\left[1+\sum_{r=1}^{2 m} \frac{1}{r !}\left(C_{1}^{\prime} K_{0} / m\right)^{r}\right] R_{m} \\
& \leq \exp \left(C_{1}^{\prime} K_{0} / m\right) \cdot R_{m} .
\end{aligned}
$$

Hence for some positive constant $C_{2}^{\prime}$, we have

$$
\left|\bar{p}_{m}(x, y)\right| \leq C_{2}^{\prime} R_{m} \quad \text { on } \quad \hat{l}_{p}(\delta) .
$$

Taking into account (7.14), we thus see that

$$
\left|\bar{p}_{m}(x, y)\right| \leq C_{3}^{\prime} \delta^{-\lambda_{2}} \cdot \max _{\bar{D}}\left|u_{m}(x, y)\right| \quad \text { on } \quad l_{p} \cap \bar{D}
$$

for some positive constant $C_{3}^{\prime}$. 
From (7.19), by the theorem of Markov, we then have

$$
\begin{aligned}
\left|\frac{d}{d s} \bar{p}_{m}(x, y)\right| & \leq C_{4}^{\prime} \delta^{-\lambda_{2}} m^{2} \cdot \max _{\bar{D}}\left|u_{m}(x, y)\right| \\
& =C_{4}^{\prime} m^{3 \lambda_{2}+2} \cdot \max _{\bar{D}}\left|u_{m}(x, y)\right| \text { on } l_{p} \cap \bar{D}
\end{aligned}
$$

for some positive constant $C_{4}^{\prime}$. Since $u_{m}(x, y)=\omega(x, y) \bar{p}_{m}(x, y)$, and $\omega(x, y), \partial_{i} \omega(x, y)(i=1,2)$ are all bounded in $\bar{D}$, taking into account (7.19), we thus see that

$$
\left|\frac{d}{d s} u_{m}(x, y)\right| \leq C_{5}^{\prime} m^{3 \lambda_{2}+2} \cdot \max _{\bar{D}}\left|u_{m}(x, y)\right| \quad \text { on } \quad l_{p} \cap \bar{D}
$$

for some positive constant $C_{5}^{\prime}$. Now by (7.11),

$$
\max _{\bar{D}}\left|u_{m}(x, y)\right| \leq M_{0}+M_{m},
$$

therefore from (7.12) and (7.20), we have

$$
\begin{aligned}
\int_{l_{\rho} \cap \bar{D}}\left(\frac{d \eta_{m}}{d s}\right)^{2} d s & \leq 2 \int_{l_{\rho} \cap \bar{D}}\left[\left(\frac{d u_{m}}{d s}\right)^{2}+\left(\frac{d u}{d s}\right)^{2}\right] d s \\
& \leq 2 K+2 C_{5}^{\prime 2} \cdot\left(M_{0}+M_{m}\right)^{2} m^{6 \lambda_{2}+4} .
\end{aligned}
$$

Inequalities (7.10) and (7.21) imply by Theorem 4.1 that

$$
M_{m} \leq A \sqrt{m^{-\alpha} \log \frac{G+H\left(M_{0}+M_{m}\right)^{2} m^{6 \lambda_{2}+4}}{\mathrm{~m}^{-\alpha}}}+B \sqrt{m^{-\alpha}}
$$

for sufficiently large $m$, where $A, B, G, H$ are suitable positive constants. Now we shall show that (7.22) implies the boundedness of $M_{m}$ for $m \geqslant m_{0}$ provided $m_{0}$ is sufficiently large. Suppose $M_{m}$ is not bounded for $m \geqslant m_{0}$. Then there is a sequence $\left\{m_{i}\right\}$ such that $m_{0} \leq$ $m_{1}<m_{2}<\cdots \rightarrow \infty$ and $M_{m} \rightarrow \infty$ as $i \rightarrow \infty$.

For brevity, let us drop suffix $i$ of $m_{i}$. Then in (7.22), we may suppose that $m \rightarrow \infty$ and $M_{m} \rightarrow \infty$. Then from (7.22) we have

$$
M_{m} \leq A\left[m^{-\alpha} \log H\left(M_{0}+M_{m}\right)^{2} m^{6 \lambda_{2}+4}(1+\sigma) m^{\alpha}\right]^{1 / 2}+B\left(m^{-\alpha}\right)^{1 / 2},
$$

where $\sigma$ is a positive constant. Then we have

$$
\begin{aligned}
1 \leq A & {\left[\frac{\log H}{m^{\alpha} \bar{M}_{m}^{2}}+\frac{2 \log \left(M_{0}+M_{m}\right)}{m^{\alpha} M_{m}^{2}}+\frac{\left(6 \lambda_{2}+4\right)}{m^{\alpha} M_{m}^{2}} \overline{\log m}\right.} \\
& \left.+\frac{\log (1+\sigma)}{m^{\alpha} M_{m}^{2}}+\frac{\alpha \log m}{m^{\alpha} M_{m}^{2}}\right]^{1 / 2}+B\left[\frac{1}{m^{\alpha} M_{m}^{2}}\right]^{1 / 2} \cdot
\end{aligned}
$$


However, the right-hand side of the above inequality evidently tends to zero as $m \rightarrow \infty$ and $M_{m} \rightarrow \infty$. This is the contradiction. Thus we see that $M_{m}$ is bounded for $m \geqslant m_{0}$, or in other words, there is a positive constant $M$ inderendent of $m$ such that

$$
M_{m} \leq M \text { for } m \geqslant m_{0} .
$$

Then (7.22) implies

$$
M_{m}=O\left[\sqrt{m^{-\alpha} \log m}\right],
$$

which completes the proof.

Q. E. D.

\section{References}

[1] Kantorovich, L. V. and V. I. Krylov, Approximate methods of higher analysis, Interscience, New York. 1958.

[2] Harrik, I. Yu., On approximation of functions vanishing on the boundary of a region by functions of a special form, Mat. Sb. 37 (1955). 353-384. (Russian)

[3] Petryshyn. W. V., Direct and iterative methods for the solution of linear operator equation in Hilbert space, Trans. Amer. Math. Soc. 105 (1962), 137-175.

[4] Mihlin. S. G., Variational methods in mathematical physics, Pergamon Press, Long Island City, 1964.

[5] Urabe, M.. Numerical solution of multi-point boundary value problems in Chebyshev series-theory of the method, Numer. Math. 9 (1967), 341-366.

[6] Nagumo, M., On principally linear elliptic differential equation of the second order, Osaka Math. J. 6 (1954), 207-229.

[7] Akô. K., On the modern and the classical solution of the Dirichlet problem, Japan. J. Math. 36 (1966), 85-97.

[8] Hobson, F. W., The theory of functions of a real variable and the theory of Fourier's series, Dover, New York, 1957.

[9] Natanson, I. P., Constructive theory of functions, II, Ungar, New York, 1964. 
